\title{
Pulsed Field Magnetization of Bridge-Seeded, Bulk YBCO using Solenoid and Split Coils
}

\author{
Mark D. Ainslie, Member, IEEE, Hiroyuki Fujishiro, Keita Takahashi, Yun-Hua Shi, and David A. Cardwell
}

\begin{abstract}
The multi-seeding process has the potential to enlarge the sample size of (RE)BCO (where RE = rare earth or Y) single-grain, bulk superconductors with improved fabrication speed, and in previous studies on multi-seeding, a significant improvement was made in the alignment of the seeds in such samples using a novel bridge-seeding technique. In this paper, we report the experimental measurements of the pulsed field magnetization (PFM) of a $0^{\circ}-0^{\circ}$ bridge-seeded Y-Ba-Cu-O (YBCO) sample. The PFM is carried out using a solenoid coil, as well as a split coil arrangement with an iron yoke, at temperature of 65,40 and $20 \mathrm{~K}$, and the resultant trapped fields and magnetic flux dynamics for these two PFM techniques are compared. It is shown that such bridge-seeded bulk YBCO can be fabricated that performs as a bulk magnet with trapped fields comparable to or better than standard, single-seeded high- $J_{c}$ samples, with the potential of enlarging the sample size. Furthermore, the split coil arrangement with an iron yoke is useful to enhance the trapped field and has a positive effect on the maximum temperature rise in the sample, which increases at lower temperatures and seriously impacts the achievable trapped field from PFM.
\end{abstract}

Index Terms-Bulk superconductors, high-temperature superconductors, magnetic flux penetration, magnetization processes, multi-seeding, pulsed field magnetization, trapped field magnets.

\section{INTRODUCTION}

$\mathrm{T}$ HE multi-seeding process has the potential to improve the fabrication speed and enlarge the sample size of (RE)BCO (where $\mathrm{RE}=$ rare earth or $\mathrm{Y}$ ) single-grain, bulk superconductors, which can trap large magnetic fields in excess of $17 \mathrm{~T}$ at temperatures below $30 \mathrm{~K}$ [1], [2] and up to $3 \mathrm{~T}$ at $77 \mathrm{~K}$ [3]. In previous studies on multi-seeding, a significant improvement was made in the alignment of the seeds in such samples using a novel bridge-seeding technique [4]-[6]. In our recent work, the pulsed field magnetization (PFM) of multi-seed samples fabricated using $45^{\circ}-45^{\circ}$ and $0^{\circ}$ $0^{\circ}$ bridge seeds was reported [7]; however, it was clear in this particular case that the seeds in the $0^{\circ}-0^{\circ}$ configuration grew

M. D. Ainslie would like to acknowledge financial support from a Royal Academy of Engineering Research Fellowship and a Royal Society International Exchanges Scheme Grant, IE131084. H. Fujishiro would like to acknowledge financial support from the Open Partnership Joint Projects of the Japan Society for the Promotion of Science (JSPS) Bilateral Joint Research Projects, and JSPS KAKENHI (23560002 and 15K04646).

M. D. Ainslie, Y-H. Shi and D. A. Cardwell are with the Bulk Superconductivity Group, Department of Engineering, University of Cambridge, Cambridge CB2 1PZ, UK (e-mail: mark.ainslie@eng.cam.ac.uk).

H. Fujishiro and K. Takahashi are with the Department of Physical Science and Materials Engineering, Faculty of Science and Engineering, Iwate University, Morioka 020-8551, Japan (e-mail: fujishiro@iwate-u.ac.jp). differently and sub-optimally, resulting in different sizes after polishing and a significantly asymmetric trapped field and magnetic flux dynamics during the PFM process. In this paper, we report the experimental measurements of a new, wellgrown $0^{\circ}-0^{\circ}$ bridge-seeded, bulk Y-Ba-Cu-O (YBCO) sample. The PFM is carried out using a solenoid coil, as well as a split coil arrangement with an iron yoke, and the resultant trapped fields and magnetic flux dynamics for these two PFM techniques are compared.

\section{SAMPLE INFORMATION}

For this study, a $0^{\circ}-0^{\circ}$ bridge-seeded, bulk YBCO sample was fabricated following the same fabrication process described in [7]. Firstly, single-grain Sm-Ba-Cu-O (SmBCO), which forms a suitable seed for the melt-processing of YBCO due to its higher melting temperature, was fabricated by topseeded melt-growth (TSMG) using a generic seed [8], [9]. The as-grown single grain was then cut into a slice of length $10 \mathrm{~mm}$, parallel to the $a$ direction of the crystallographic lattice, which was then machined into a bridge-shaped seed and used to multi-seed the bulk YBCO sample based on controlled alignment and relative orientation of the two legs of the seed $\left(0^{\circ}-0^{\circ}\right)$ [4]. Special care was paid to the alignment of the seeds [10] by selecting a better bridge seed by visual examination, as commonly carried out when selecting single seeds for normal TSMG.

Precursor powders of $75 \mathrm{wt} \% \mathrm{YBa}_{2} \mathrm{Cu}_{3} \mathrm{O}_{7}$ (Y-123), $25 \mathrm{wt} \%$ $\mathrm{Y}_{2} \mathrm{BaCuO}_{5}(\mathrm{Y}-211)$ and $0.5 \mathrm{wt} \% \mathrm{CeO}_{2}(99.9 \%$ purity) were mixed thoroughly using a motorized mortar and pestle [6]. The mixed powder was pressed uniaxially into a pellet and the carefully chosen bridge seed was placed on the top surface of the pellet. The TSMG technique was used to fabricate the sample, which involves heating the pellet to $1045^{\circ} \mathrm{C}$, holding for $1 \mathrm{~h}$, cooling at $100^{\circ} \mathrm{C} / \mathrm{h}$ to $1005^{\circ} \mathrm{C}$, and then cooling more slowly at $0.4^{\circ} \mathrm{C} / \mathrm{h}$ to $970^{\circ} \mathrm{C}$. Finally, the sample was furnacecooled down to room temperature at $200^{\circ} \mathrm{C} / \mathrm{h}$. The multi-seed sample, after machining and polishing, is $26.0 \mathrm{~mm}$ in diameter and $13.2 \mathrm{~mm}$ in height.

A photograph of the top surface of the sample is shown in Fig. 1 (left) and the sample mounted in the copper sample holder for PFM experiments using the split coil arrangement is shown in Fig. 1 (right), including a thin ring of indium to ensure a good thermal contact. The 2D trapped field distributions presented throughout the paper correspond to the same alignment shown in Fig. 1. The peak trapped field of the sample, obtained by field cooling (FC) magnetization at $77 \mathrm{~K}$ in an external magnetic field of $1.5 \mathrm{~T}$, was measured using a 
hand probe: $B_{\mathrm{T}}(\mathrm{FC})=0.79 \mathrm{~T}$ (top surface), $B_{\mathrm{T}}(\mathrm{FC})=-0.59 \mathrm{~T}$ (bottom surface).
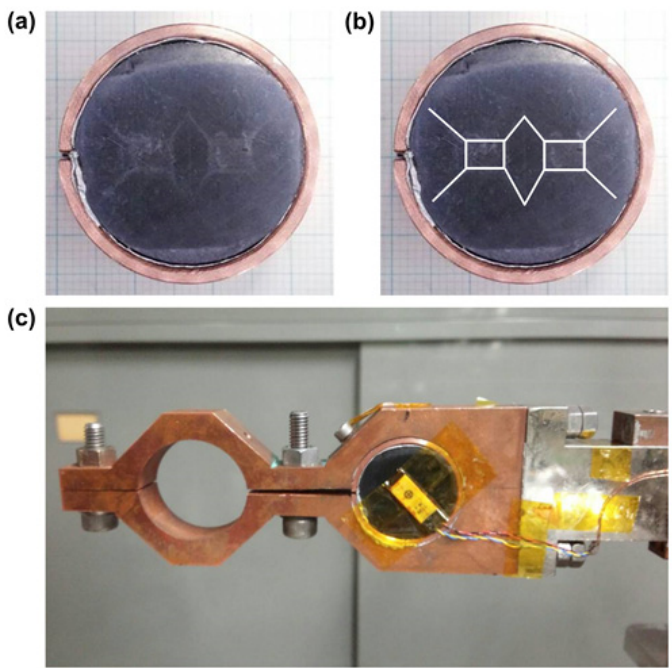

Fig. 1. (a) Photograph of the top surface of the $0^{\circ}-0^{\circ}$ bridge-seeded YBCO bulk sample, including a thin ring of indium and copper ring around the periphery of the sample to ensure a good thermal contact. (b) Location of the $0^{\circ}-0^{\circ}$ bridge seed and growth sector boundaries (GSBs) shown by solid white lines. (c) Sample mounted in the sample holder for PFM experiments using the split coil arrangement.

\section{Pulsed Field Magnetization Results}

\section{A. Split Coil Pulsed Field Magnetization}

An overview of the split coil PFM experimental setup is described in [11], [12]. The sample was fastened in a copper holder with thin indium foil $(0.2 \mathrm{~mm}$ in thickness) and connected to the cold stage of a Gifford-McMahon cycle, helium refrigerator in the vacuum chamber. A Hall sensor (BHT 921; F W Bell) was adhered to the center of the bulk surface, and a Cernox ${ }^{\mathrm{TM}}$ thermometer was connected to the copper holder. The split coil has an inner diameter of $72 \mathrm{~mm}$, an outer diameter of $124 \mathrm{~mm}$ and a height of $35 \mathrm{~mm}$, and is submerged in liquid nitrogen outside the vacuum chamber. A pair of soft iron, Ni-plated yokes $(60 \mathrm{~mm}$ in diameter and $65 \mathrm{~mm}$ in height) can be inserted in the central bores of the coil, which was shown to enhance the trapped field in bulk high-temperature superconductors [11], as well as bulk $\mathrm{MgB}_{2}$ [12]. The initial temperature of the bulk was set to $T_{\mathrm{s}}=65,40$ and $20 \mathrm{~K}$, and magnetic pulses, $B_{\mathrm{ex}}$, up to around $6 \mathrm{~T}$, with a rise time of $t_{\mathrm{r}}=18 \mathrm{~ms}$ and duration of approximately $t_{\mathrm{d}}=$ $200 \mathrm{~ms}$ were applied via a pulse current in the coil.

Fig. 2 shows a comparison of the measured trapped fields at approximately $t=300 \mathrm{~ms}$ after activation by PFM using the split coil with and without the iron yoke. The split coil with the iron yoke results in a significantly higher trapped field, but a slightly higher activation field (the field required to fully magnetize the sample [13]). It should be noted that these results include the presence of the iron yoke; two-dimensional (2D) trapped field distributions (Figs. 5-8) were also measured about $t=20 \mathrm{~min}$ after application of the pulse with the split coil (and iron yoke) removed, by scanning a Hall sensor using an $x-y$ stage controller on the outside surface of the vacuum chamber at a distance approximately $2 \mathrm{~mm}$ above the bulk surface. Using these 2D trapped field distributions, the total trapped flux in the sample was calculated, as shown in Fig. 3. It is clear that more flux is trapped when the iron yoke is inserted into the split coil during the PFM process.

Fig. 4 shows the temperature rise in the sample during the PFM process with and without the iron yoke as measured by the Cernox ${ }^{\mathrm{TM}}$ thermometer. For lower temperatures, in particular, the presence of the iron yoke slightly reduces the temperature rise, which is important for practical applications because the temperature rise limits the trapped field achievable with PFM [14]. The much higher temperature rise when $T_{\mathrm{s}}=20 \mathrm{~K}$ severely limits the trapped field performance and results in similar trapped fields/flux to that at $T_{\mathrm{s}}=40 \mathrm{~K}$ because of this.

Fig. 5 shows the 2D trapped field distribution when the sample is partially magnetized, which helps understand the magnetic flux dynamics during the PFM process and the $J_{\mathrm{c}}$ distribution within the sample [13], for the case where the iron yoke is inserted into the split coil $\left(B_{\mathrm{ex}}=3.68 \mathrm{~T}, T_{\mathrm{s}}=40 \mathrm{~K}\right)$. Fig. 6 shows the same plot for the case where the iron yoke is not inserted $\left(B_{\mathrm{ex}}=3.33 \mathrm{~T}, T_{\mathrm{s}}=40 \mathrm{~K}\right)$. In the partially magnetized case, the flux appears to be trapped close to the growth sector boundaries (GSBs) seen in Fig. 1 (left), consistent with the results presented in [13] for a standard, single-seeded YBCO sample, and the location of this trapped flux is opposite to that observed in the partially magnetized cases for the $45^{\circ}-45^{\circ}$ bridge-seeded sample presented in [7].

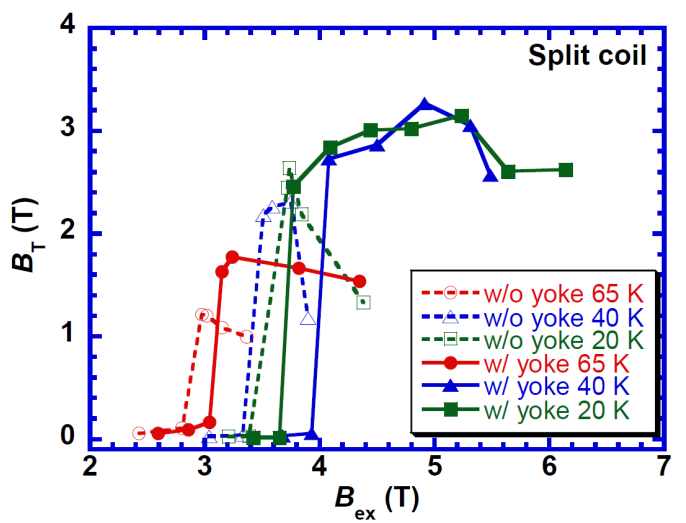

Fig. 2. Comparison of trapped fields at the centre of the top surface of the $0^{\circ}$ $0^{\circ}$ bridge-seeded, bulk YBCO sample after pulsed field magnetization with the split coil with an iron yoke inserted (solid lines) and without (dashed lines) at operating temperatures of 65,40 and $20 \mathrm{~K}$.

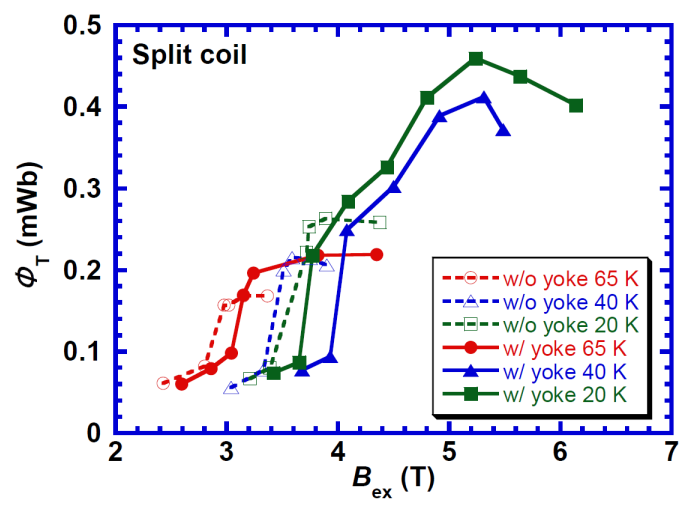

Fig. 3. Comparison of trapped flux in the $0^{\circ}-0^{\circ}$ bridge-seeded, bulk YBCO sample after pulsed field magnetization with the split coil with an iron yoke inserted (solid lines) and without (dashed lines) at operating temperatures of 65,40 and $20 \mathrm{~K}$. 


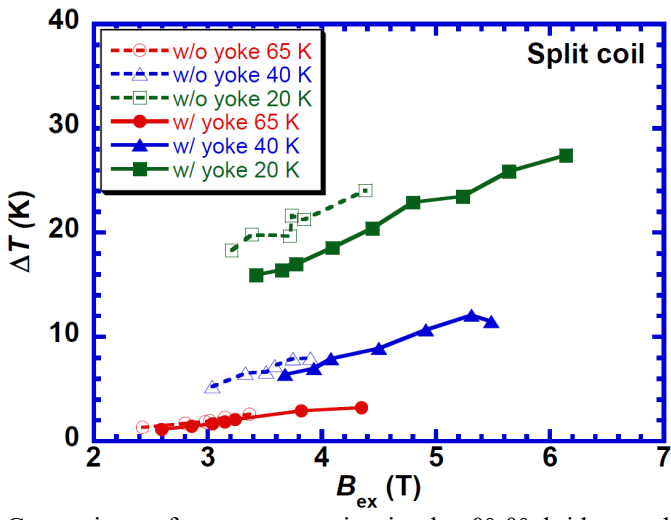

Fig. 4. Comparison of temperature rise in the $0^{\circ}-0^{\circ}$ bridge-seeded, bulk YBCO sample during pulsed field magnetization with the split coil with an iron yoke inserted (solid lines) and without (dashed lines) at operating temperatures of 65,40 and $20 \mathrm{~K}$.

Figs. 7 and 8 show the 2D trapped field distributions when the sample is fully magnetized, for the case where the iron yoke is inserted $\left(B_{\mathrm{ex}}=4.92 \mathrm{~T}, T_{\mathrm{s}}=40 \mathrm{~K}\right)$ and not inserted $\left(B_{\mathrm{ex}}\right.$ $\left.=3.75 \mathrm{~T}, T_{\mathrm{s}}=40 \mathrm{~K}\right)$, respectively. The trapped field exhibits a traditional conical profile $\left(B_{\mathrm{t}} \approx 1.5 \mathrm{~T}\right.$ (with yoke), $B_{\mathrm{t}} \approx 0.9 \mathrm{~T}$ (without yoke)), although twin peaks in the trapped field profile due to the $0^{\circ}-0^{\circ}$ bridge seed could be expected if measured much closer to the surface, depending on the depth of the grain boundary formed between the seeds. This profile suggests this grain boundary is very shallow. The trapped fields indicate that the sample performs as a bulk magnet comparably to or better than standard, single-seeded high- $J_{\mathrm{c}}$ samples [11], [15], with the potential of larger sample sizes.

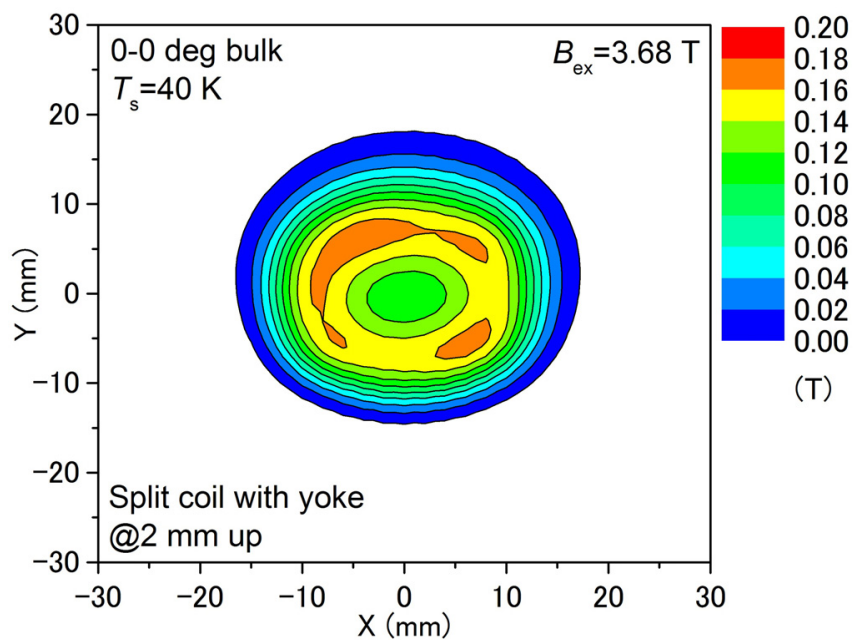

Fig. 5. 2D trapped field profile measured $2 \mathrm{~mm}$ above the top surface of the sample after PFM at $40 \mathrm{~K}$ using the split coil with an iron yoke, for an applied field $B_{\mathrm{ex}}=3.68 \mathrm{~T}$. This resulted in partial magnetization of the sample.

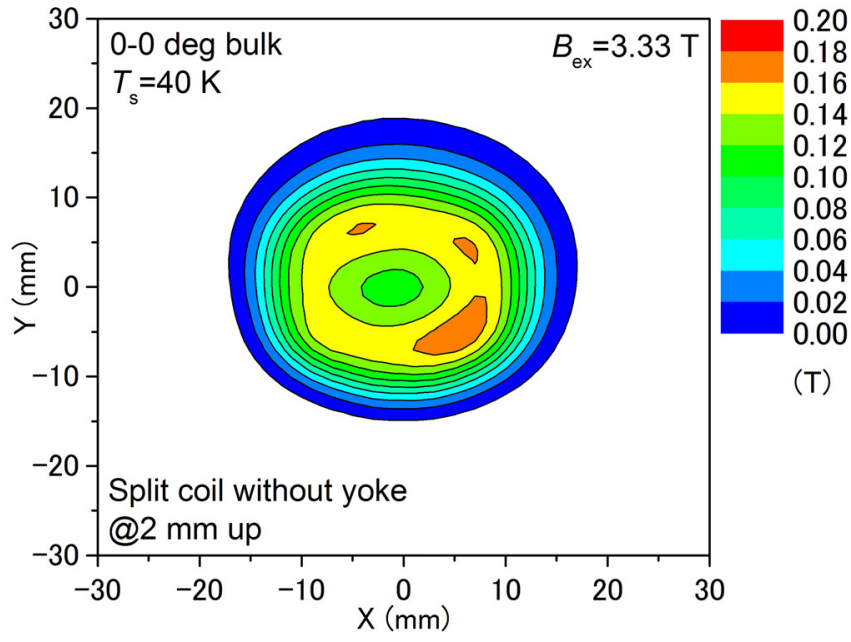

Fig. 6. 2D trapped field profile measured $2 \mathrm{~mm}$ above the top surface of the sample after PFM at $40 \mathrm{~K}$ using the split coil without an iron yoke, for an applied field $B_{\mathrm{ex}}=3.33 \mathrm{~T}$. This resulted in partial magnetization of the sample.

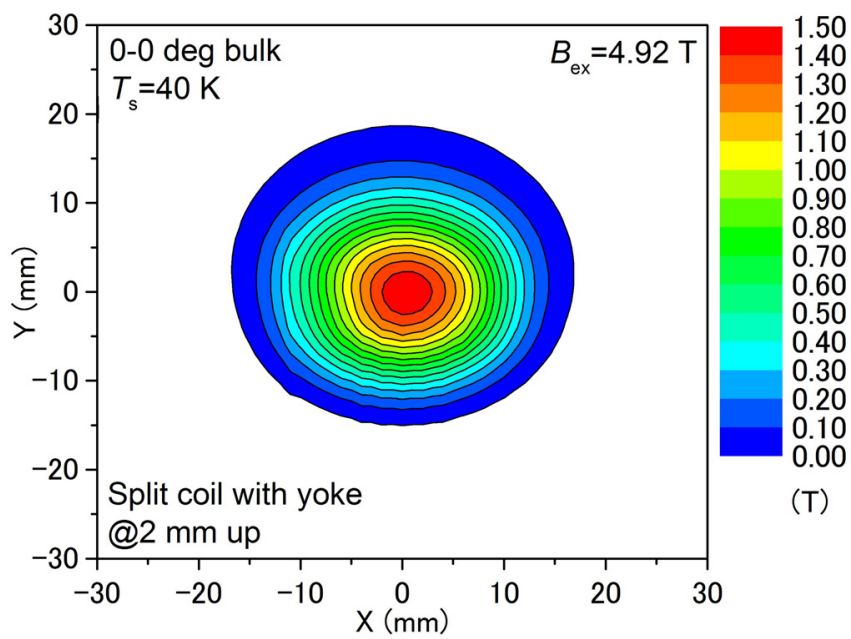

Fig. 7. 2D trapped field profile measured $2 \mathrm{~mm}$ above the top surface of the sample after PFM at $40 \mathrm{~K}$ using the split coil with an iron yoke, for an applied field $B_{\mathrm{ex}}=4.92 \mathrm{~T}$. This resulted in full magnetization of the sample.

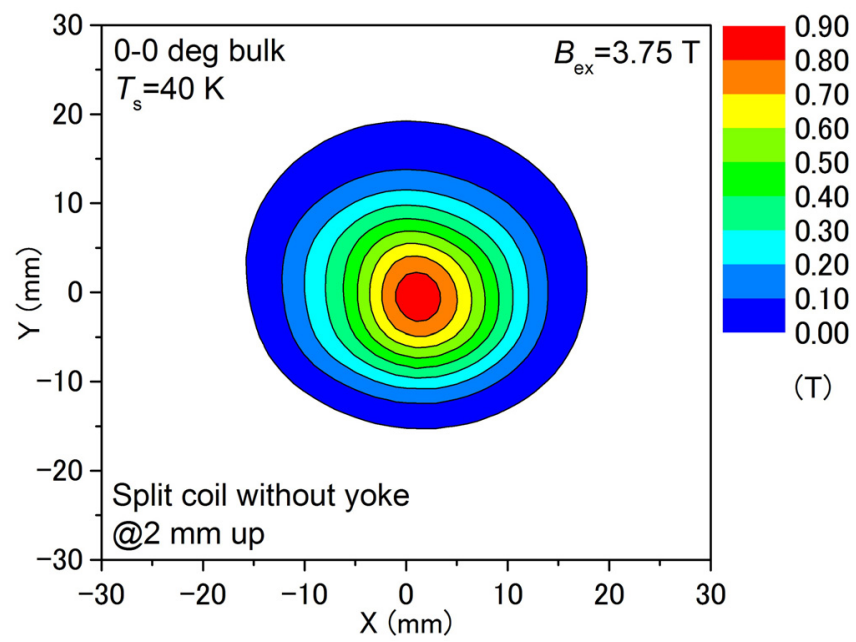

Fig. 8. 2D trapped field profile measured $2 \mathrm{~mm}$ above the top surface of the sample after PFM at $40 \mathrm{~K}$ using the split coil without an iron yoke, for an applied field $B_{\mathrm{ex}}=3.75 \mathrm{~T}$. This resulted in full magnetization of the sample. 


\section{B. Solenoid Coil Pulsed Field Magnetization}

An overview of the solenoid coil PFM experimental setup is described in [13]. The bulk sample was mounted tightly using a sample holder fabricated from 316 stainless steel, of inner diameter slightly larger than the diameter of the samples and outer diameter $56 \mathrm{~mm}$, to match the dimensions of the available cold stage of the pulse system. Stycast ${ }^{\mathrm{TM}}$ was used to mount the samples in the holder, which was then mounted on the cold stage of a Gifford-McMahon (GM), closed cycle helium refrigerator, and a copper magnetizing solenoid pulse coil, cooled using liquid nitrogen, was placed outside the vacuum chamber. The direction of external applied field is parallel to the $c$-axis of the bulk samples. The solenoid magnetizing coil can provide pulsed external fields up to $B_{\text {ex }}=$ $5.4 \mathrm{~T}$, with a rise time of $t_{\mathrm{r}}=12 \mathrm{~ms}$ and duration of approximately $t_{\mathrm{d}}=120 \mathrm{~ms}$. In this setup, an iron yoke is embedded in the magnetizing fixture, as shown in [11], which is present for all of the following measurements.

The trapped field close to the centre of the top surface of the sample was measured dynamically during each pulse using a Hall sensor, and 2D trapped field distributions were measured inside the vacuum chamber using an $x-y$ stage controller and the same Hall sensor, approximately $2 \mathrm{~mm}$ above the top surface.

Fig. 9 shows a comparison of the measured trapped fields at approximately $t=300 \mathrm{~ms}$ after activation by PFM using the solenoid coil and the split coil with the iron yoke. The solenoid coil results in a significantly lower trapped field than the split coil with the iron yoke, as well as a higher activation field, which is particularly noticeable when $T_{\mathrm{s}}=40$ and $20 \mathrm{~K}$.

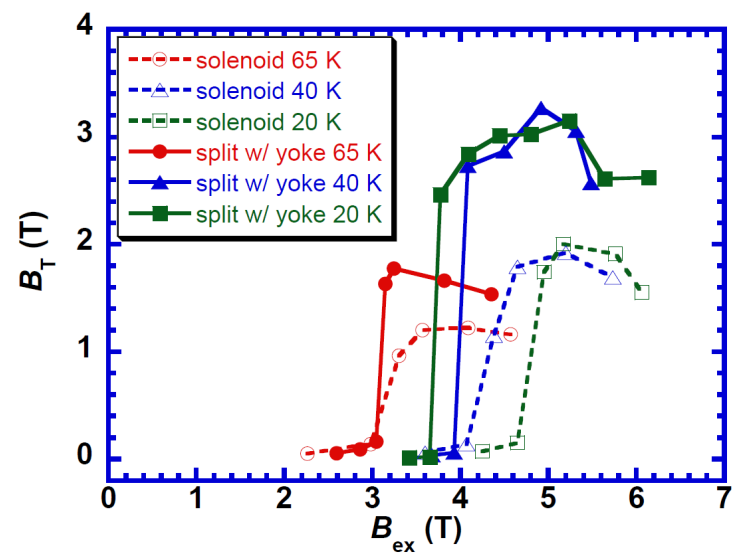

Fig. 9. Comparison of trapped fields at the centre of the top surface of the $0^{\circ}$ $0^{\circ}$ bridge-seeded, bulk YBCO sample after pulsed field magnetization with a solenoid coil (dashed lines) and the split coil with an iron yoke (solid lines) at operating temperatures of 65,40 and $20 \mathrm{~K}$.

Fig. 10 shows the 2D trapped field distribution when the sample is partially magnetized using the solenoid coil $\left(B_{\mathrm{ex}}=\right.$ $\left.3.60 \mathrm{~T}, T_{\mathrm{s}}=40 \mathrm{~K}\right)$. As found for the split coil in the previous section, the flux appears to be trapped close to the GSBs and the location of this trapped flux is opposite to that observed in the partially magnetized cases for the $45^{\circ}-45^{\circ}$ bridge-seeded sample presented in [7].

Fig. 11 shows the $2 \mathrm{D}$ trapped field distribution when the sample is fully magnetized using the solenoid coil $\left(B_{\mathrm{ex}}=\right.$ $\left.5.20 \mathrm{~T}, T_{\mathrm{s}}=40 \mathrm{~K}\right)$. Again, the trapped field exhibits a traditional conical profile $\left(B_{\mathrm{t}} \approx 1.3 \mathrm{~T}\right)$, but the magnitude of the trapped field is lower than that obtained using the split coil with an iron yoke. As found for the PFM results for the split coil with an iron yoke, the trapped field is comparable to or better than that achieved with the same setup for standard, single-seeded high- $J_{\mathrm{c}}$ samples [11], [15].

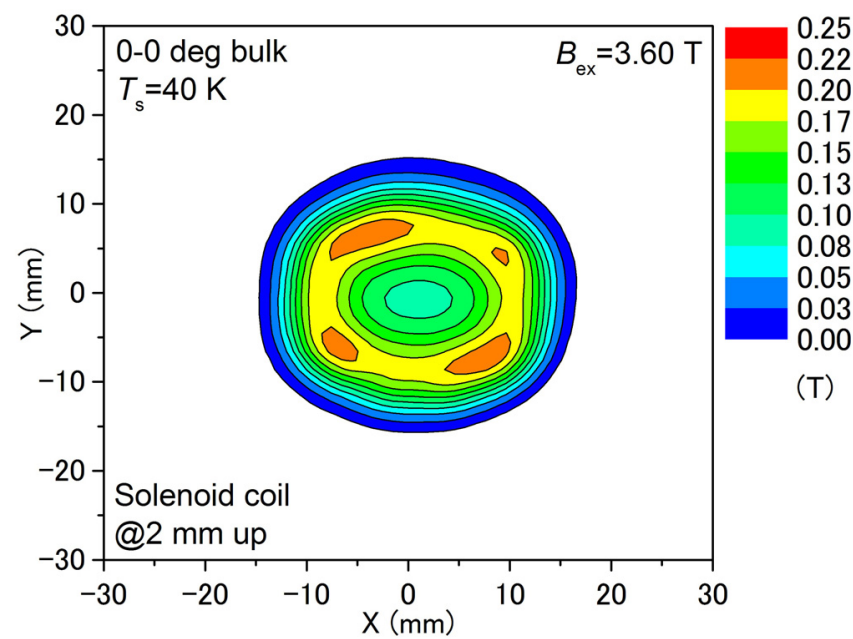

Fig. 10. 2D trapped field profile measured $2 \mathrm{~mm}$ above the top surface of the sample after PFM at $40 \mathrm{~K}$ using the solenoid coil, for an applied field $B_{\text {ex }}=$ $3.60 \mathrm{~T}$. This resulted in partial magnetization of the sample.

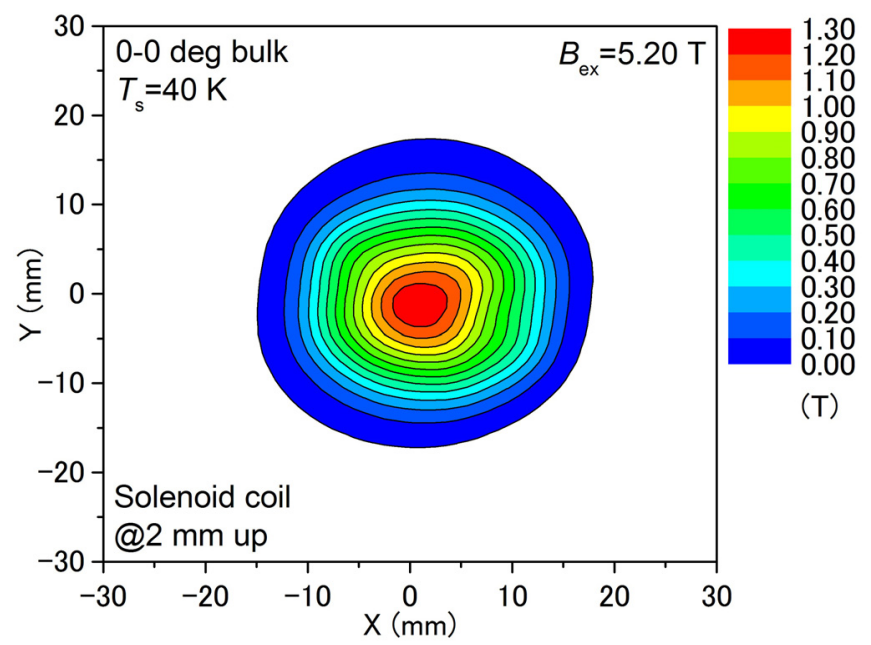

Fig. 11. 2D trapped field profile measured $2 \mathrm{~mm}$ above the top surface of the sample after PFM at $40 \mathrm{~K}$ using the solenoid coil, for an applied field $B_{\text {ex }}=$ $5.20 \mathrm{~T}$. This resulted in full magnetization of the sample.

\section{CONCLUSION}

In this paper, the experimental measurements of the PFM of a $0^{\circ}-0^{\circ}$ bridge-seeded, bulk Y-Ba-Cu-O (YBCO) sample were carried out using a solenoid coil, as well as a split coil arrangement with and without an iron yoke. The resultant trapped fields and magnetic flux dynamics for these two PFM techniques are compared, with a number of important findings:

1) $0^{\circ}-0^{\circ}$ bridge-seeded bulk YBCO can be fabricated, by carefully selecting an appropriate bridge seed to control the alignment of the seeds, that performs as a bulk magnet with 
trapped fields and flux comparable to or better than standard, single-seeded high- $J_{\mathrm{c}}$ samples [11], [15], with the potential of enlarging the sample size.

2) When the sample is partially magnetized, magnetic flux appears to be trapped close to the GSBs and the location of this trapped flux is opposite to that observed in the partially magnetized cases for the $45^{\circ}-45^{\circ}$ bridge-seeded sample presented previously [7].

3) There is a much higher temperature rise for lower operating temperatures, which severely limits the trapped field performance. When $T_{\mathrm{s}}=20 \mathrm{~K}$, the magnitude of the trapped field/flux is similar to that observed at $T_{\mathrm{s}}=40 \mathrm{~K}$ because of this.

4) A split coil arrangement with an iron yoke can enhance the trapped field in bulk superconductors in comparison to a conventional split coil (without an iron yoke) or solenoid coil, and the use of the iron yoke appears to have a positive effect on the maximum temperature rise in the sample, which increases at lower temperatures.

\section{REFERENCES}

[1] M. Tomita, and M. Murakami, "High-temperature superconductor bulk magnets that can trap magnetic fields of over 17 tesla at $29 \mathrm{~K}$," Nature, vol. 421, pp. 517-520, Jan. 2003.

[2] J. H. Durrell et al., "A trapped field of $17.6 \mathrm{~T}$ in melt-processed, bulk Gd-Ba-Cu-O reinforced with shrink-fit steel," Supercond. Sci. Technol., vol. 27, no. 8, Aug. 2014, Art. ID. 082001.

[3] S. Nariki, N. Sakai, and M. Murakami, "Melt-processed Gd-Ba-Cu-O superconductor with trapped field of $3 \mathrm{~T}$ at $77 \mathrm{~K}$," Supercond. Sci. Technol., vol. 18, no. 2, pp. S126-S130, Feb. 2005.

[4] Y-H. Shi, J. H. Durrell, A. R. Dennis, and D. A. Cardwell, "Bulk YBCO seeded with $45^{\circ}-45^{\circ}$ bridge-seeds of different lengths," Supercond. Sci. Technol., vol. 26, no. 1, Jan. 2013, Art. ID. 015012.

[5] Y-H. Shi et al., "Properties of grain boundaries in bulk, melt processed Y-Ba-Cu-O fabricated using bridge-shaped seeds," Supercond. Sci. Technol., vol. 25, no. 4, Apr. 2012, Art. ID. 045006.

[6] Y-H. Shi et al., "A Comparison of $0^{\circ}-0^{\circ}$ and 45-45 Bridge-Seeded, YBCO Single Grains,” J. Am. Ceram. Soc., vol. 96, no. 6, pp. 1757-62, Jun. 2013.

[7] M. D. Ainslie et al., "Pulsed field magnetization of $0^{\circ}-0^{\circ}$ and $45^{\circ}-45^{\circ}$ bridge-seeded Y-Ba-Cu-O bulk superconductors," Supercond. Sci. Technol., vol. 28, no. 12, Dec. 2015, Art. ID. 125002.

[8] Y. Shi, N. Hari Babu, and D. A. Cardwell, "Development of a generic seed crystal for the fabrication of large grain (RE)-Ba-Cu-O bulk superconductors," Supercond. Sci. Technol., vol. 18, no. 4, pp. L13-L16 Apr. 2005.

[9] N. Hari Babu, Y. Shi, K. Iida, and D. A. Cardwell, "A practical route for the fabrication of large single-crystal (RE)-Ba-Cu-O superconductors," Nat. Mater., vol. 4, pp. 476-480, Jun. 2005.

[10] Y. Shi et al., "Factors Affecting the Growth of Multiseeded Superconducting Single Grains" Cryst. Growth Des., vol. 16, pp. 5110-5117, Jul. 2016

[11] M. D. Ainslie et al., "Enhanced trapped field performance of bulk hightemperature superconductors using split coil, pulsed field magnetization with an iron yoke," Supercond. Sci. Technol., vol. 29, no. 7, Jul. 2016, Art. ID. 074003.

[12] H. Fujishiro, H. Mochizuki, M. D. Ainslie, and T. Naito, "Trapped field of $1.1 \mathrm{~T}$ without flux jumps in an $\mathrm{MgB}_{2}$ bulk during pulsed field magnetization using a split coil with a soft iron yoke," Supercond. Sci. Technol., vol. 29, no. 8, Aug. 2016, Art. ID. 084001.

[13] M. D. Ainslie et al., "Modelling and comparison of trapped fields in (RE)BCO bulk superconductors for activation using pulsed field magnetization," Supercond. Sci. Technol., vol. 27, no. 6, Jun. 2014, Art. ID. 065008 .

[14] M. D. Ainslie and H. Fujishiro, "Modelling of bulk superconductor magnetization," Supercond. Sci. Technol., vol. 28, no. 5, May 2015, Art ID. 053002 .
[15] M. D. Ainslie et al., "Pulsed Field Magnetization of Single-Grain Bulk YBCO Processed From Graded Precursor Powders," IEEE Trans. Appl. Supercond., vol. 26, no. 4, Jun. 2016, Art. ID. 6800104. 